\title{
Grammatik, Höflichkeit und Gender in der Zweiten Person. Über Anrede-Genus*
}

Horst J. Simon (Berlin)

\begin{abstract}
In this paper, I study various manifestations of the grammatical category gender in addressrelated forms, above all in second person pronouns. It will be shown that its use is intricately connected with politeness - either because the gendered pronouns of address are themselves polite forms or because they can, under certain circumstances, convey nuances of social gender and concomitant connotations. The languages studied include, among others, Japanese, Arabic and other Semitic languages, as well as historical and dialectal varieties of German and Italian.
\end{abstract}

\section{Genus als variable grammatische Kategorie}

Studienanfänger*innen wird gern erzählt, dass die grammatischen Kategorien einigermaßen klar verteilt seien: Kasus ist eine nominale Kategorie und wird dementsprechend - wenn er denn überhaupt morphologisch zum Tragen kommt - an Artikeln, Nomina usw. markiert; Tempus ist eine rein verbale Kategorie und wird deshalb an Verben markiert; und Person und Numerus sind zwei Kategorien, die meist gemeinsam auftreten und die sowohl im verbalen als auch im pronominalen Bereich paradigmenbildend wirken können (in ersterem typischerweise affixal, in letzterem meist suppletiv). Bei genauerem Hinsehen erweist sich eine solche Darstellung aber - wie eigentlich immer in der Linguistik - als nur in einem idealisierenden Sinne richtig. So zeigen Nordlinger/Sadler (2004), dass in einer Reihe von genetisch und geographisch unabhängigen Sprachen auch Nomina systematisch nach Tempus flektieren. ${ }^{1}$ Und mehrere kontinentalwestgermanische Dialekte kennen die sogenannten ,flektierenden Konjunktionen', die Person/Numerus markieren (cf. Weiß 2005 für einen Überblick). ${ }^{2}$

\footnotetext{
* Ich danke den Teilnehmer*innen der Tagung in Freiburg i. Ü. sowie den Herausgeber*innen dieses Sonderhefts sowie einer anonymen Review-Person für ihre Anregungen und Kommentare sowie insbesondere den Kolleg*innen, die mir Hinweise gegeben haben, sowie den Informant*innen, die meine ihnen oft unsinnig erscheinenden Fragen zu ihren Sprachen mit Verwunderung und Geduld ertragen haben

${ }^{1}$ Diese Analyse ist allerdings nicht unwidersprochen geblieben, cf. Tonhauser (2008); cf. nunmehr Bertinetto (2020) für eine Erweiterung der Perspektive auch auf andere ,verbale' Kategorien bei Nomina.

${ }^{2}$ Der genannte Sprachraum scheint überhaupt anfällig für Person/Numerus-Ausbreitung zu sein, was sich daran ermessen lässt, dass selbst ,Partikeln` wie das Rückversicherungselement gell im Bairischen (cf. Simon 2003:183185) oder Antwortpartikeln (JA/NEIN) in niederländischen Dialekten (cf. De Vogelaer/van der Auwera 2011) entsprechend flektieren können.
}

Linguistik online 107, 2/21 - http://dx.doi.org/10.13092/lo.107.7688

CC by 3.0 
Bei der in diesem Sonderheft im Zentrum stehenden Kategorie Genus scheint es zunächst auch klar zu sein: Genus ist nach traditioneller Auffassung eine den Nomina inhärente Eigenschaft, es wird also lexikalisch zugewiesen. Daneben taucht es an weiteren Elementen auf, bei denen ihr Wert aufgrund von Kongruenzrelationen determiniert wird - so die gängige Definition seit Hockett (1958: 231); cf. dazu auch Corbett (1991). Zu diesen Elementen zählen potentiell alle attributiv oder anaphorisch in Beziehung zu Nomina auftauchenden Wortarten: Artikel, Adjektive und (Personal)Pronomina der 3. Person, also beispielsweise auch Demonstrativ- und Relativpronomina, aber auch darüber hinausgreifend - durchaus seltener - Verben und andere Wortarten.

Nun zeigen die Arbeiten in diesem Band, dass diese traditionelle Genus-Konzeption nicht in ihrer Schlichtheit beibehalten werden kann: Spätestens seit Christen (1998) ist deutlich geworden, dass in manchen Varietäten des Deutschen sowie im Luxemburgischen genusflektierende Wörter bei Referenz auf weibliche Personen zumindest in der Kongruenz mit Controllern in Form von Eigennamen systematisch variieren, und zwar zum Ausdruck von im Wesentlichen pragmatischen Funktionen, die mit Ausdrucksbedürfnissen wie ,emotionale Nähe/Distanz', aber auch ,Mitleid' usw. umschrieben werden können. ${ }^{3}$ Ein weiterer Bereich, bei dem sich die Genus-Zuweisung als variabel darstellt, sind Hybridnomina, konkret: Personenbezeichnungen mit der - traditionell gesprochen - Genus-Spezifikation Neutrum, die sich auf weibliche Personen beziehen. Hier können neben grammatischen Faktoren, wie Wortart oder Abstand der involvierten Wörter im Satz, auch eine ganze Reihe von Kontextfaktoren die Wahrscheinlichkeit erhöhen, dass manche Kongruenz-Targets als Feminin auftauchen, wie Hübner (in diesem Band) demonstriert; sie identifiziert in diesem Zusammenhang Konnotationen wie sexuelle Relevanz der Referenz-Person oder erotische Aufladung der dargestellten Situation als maßgeblich. ${ }^{4}$

In meinem Beitrag soll nun ein m. W. bislang noch nicht im Kontext von Genus-Variation betrachteter Fall in den Vordergrund gerückt werden: eine Genus-Differenzierung, die in Zusammenhang mit Adressaten-Gender variiert. Dabei wird es um genusdifferenzierte Anredepronomina und deren Verwendungsbedingungen gehen, aber auch um andere grammatische Felder, bei denen die Kategorie ,Geschlecht‘ eine Rolle bei der Formulierung von Anrede und Verwandtem spielt. Angesichts des explorativen Charakters dieser Ausführungen soll weder Vollständigkeit noch analytische Tiefe angestrebt werden. Vielmehr soll durch das Zusammentragen von Beobachtungen aus der allgemein-typologischen Literatur, aus Beschreibungen einzelner Sprachen - soweit überhaupt vorhanden - und aus punktuellen eigenen Nachbohrungen aufgezeigt werden, mit was für einem Spektrum an Formen und Funktionsweisen zu rechnen

\footnotetext{
${ }^{3}$ Einen Überblick zur geographischen Verteilung und zu Grammatik und Pragmatik dieser Konstruktion liefert Nübling/Busley/Drenda (2013). Für detailliertere Beobachtungen zu dieser Thematik cf. jetzt die Studie von Busley/Fritzinger (in diesem Band).

4 Von der wohl nicht haltbaren Annahme von Leiss (1997) unter Rückgriff auf ältere Auffassungen, dass im Urindogermanischen, noch reflektiert bis ins Althochdeutsche, das nominale Genus quasi mit einem semantischen Wert belegt und somit relativ frei variabel gewesen sei, mithin als Shifter im Sinne Jakobsons ([1957]1971) fungiert habe, sei einmal abgesehen. Die diesbezüglich eher magere Evidenz diskutiert eingehend Froschauer (2003).
} 
ist. Eine eingehendere Untersuchung, die auf saubererer Empirie basiert, wäre wünschenswert, wenngleich nicht unaufwändig. ${ }^{5}$

Bevor eine Umschau einiger interessanter Phänomene angegangen werden kann, ist mit Rücksicht auf die Thematik dieses Beitrags eine terminologische Bemerkung angebracht: Im Folgenden werden grammatikalische Abstraktionen wie die Gesprächsrollen ,Sprecher‘ und ,Adressat' ausschließlich mit maskulinen Formen bezeichnet, während bei konkret personenreferentiellen Ausdrücken in Fällen intendierter Geschlechtsneutralität die Formen mit GenderSternchen zur Anwendung kommen. Da in der deutschen Wissenschaftssprache mit ,Genus“ und ,Gender' zwei Begriffe zur Verfügung stehen, die eine Unterscheidung von der Grammatik einerseits und dem sozialen Konstrukt des Geschlechts (im Sinne von Judith Butler) andererseits erlauben, werden diese beiden Termini auch so verwendet. Der andernorts häufig verwendete Begriff ,Sexus ‘ findet hier keine Anwendung, denn die basale biologische Verfasstheit des Menschen - wie immer man die auch definieren wolle - spielt bei der sprachlichen Kodierung von Geschlecht wohl keine Rolle; sprachlich behandelt (und natürlich erst recht verhandelt) wird stets Butler-Gender. Es wird sich zeigen, dass die Reibung zwischen verschiedenen Konzeptualisierungen des Genders von Referenz-Personen sich auch in sprachlicher Variabilität äußert.

\section{Allgemeine Differenzierungen in Bezug auf Sprecher- bzw. Adressaten-Gender}

Zunächst ist darauf hinzuweisen, dass in manchen Kulturen das sprachliche System generell nach Sprecher-Gender differiert (cf. für einen Überblick z. B. Aikhenvald 2016: 136-165): Unabhängig von anatomisch bedingten Unterschieden der Sprechwerkzeuge, die zu phonetischer Variation - in erster Linie im Hinblick auf Tonhöhen - führen (cf. überblickshaft Kotthoff/Nübling 2018: 53-59), gelten für als weiblich und für als männlich gelesene Sprecher*innen teils unterschiedliche grammatische Regeln, vor allem in der Phonologie. Aber auch syntaktische Besonderheiten gibt es manchmal: So wird ein gewisser Typ von Kopula-Deletion im Japanischen offenbar nur von weiblichen Sprecher*innen angewendet (cf. Shibatani 1990: 373). ${ }^{6}$ In vielen Gesellschaften herrschen (meist nur für Personen des einen oder des anderen Geschlechts) recht strenge Regeln im Hinblick auf die Verwendung gewisser lexikalischer Elemente, wodurch sogenannte Avoidance Registers entstehen, die große (bzw. alle?) Teile des Wortschatzes betreffen können (cf. Fleming 2014, insbesondere die Übersichtstabelle S. 126). Im Extremfall führt ein solches Verfahren also zu systematisch vollkommen unterschiedlichen Sprachformen je nach Sprecher-Gender.

Neben diesen gewissermaßen das gesamte Sprachsystem betreffenden Unterschieden finden sich sprachliche Differenzen im Hinblick auf Sprecher/Adressaten-Gender aber auch generell in vielen Sprachen, wenn auch nur in weniger stark ausgeprägtem Maße. Es handelt sich dabei oft um höflichkeitsrelevante Routineformeln im Bereich der Dankesformeln oder der Grüße.

\footnotetext{
${ }^{5}$ Da nicht wenige der einschlägigen Sprachen zu den weniger erforschten zählen, kann häufig nur auf relativ oberflächenorientierte grammatische Beschreibungen, die keine Aussagen zu soziolinguistisch relevanter Variation enthalten, zurückgegriffen werden; tiefergehende Analysen zu den womöglich variablen Gebrauchsbedingungen der Formen würden größeren empirischen Aufwand erfordern, der an dieser Stelle nicht geleistet werden kann. 6 Dass auch die beiden letzten Sätze mit einem Körnchen Unwahrheit zu nehmen sind, verdeutlicht übrigens ein vorsichtiger Blick in Motschenbacher (2007) und Azul (2013) bzw. bereits Okamoto (1995).
} 
Das Thailändische beispielsweise kodiert Sprecher-Gender in den im Alltagsdiskurs praktisch allgegenwärtigen satzfinalen Höflichkeitspartikeln, so dass die Gender-Identität der Gesprächsteilnehmer*innen fortwährend versprachlicht wird (cf. Iwasaki/Ingkaphirom 2005: 179; dort auch weitere Allomorphe): ${ }^{7}$

(1) MASK: khráp

(2) FEM: khâ

Eine ähnliche Beobachtung, nur diesmal mit Bezug auf die Gender-Identität des Adressaten, ist im Französischen Frankreichs zu beobachten, wo die traditionellen Grußformeln gegenüber Unbekannten in der Öffentlichkeit erst durch Hinzufügung eines genderkodierenden vokativischen Nomens akzeptabel werden:

(3) MASK: Bonjour, Monsieur.

(4) FEM: Bonjour, Madame.

Ein weiteres Beispiel für eine gendersensitive Routineformel, diesmal die Standard-Dankesformel, liefert das Portugiesische, hier sogar - bedingt durch die Etymologie, es handelt sich um alte Partizipialformen - in flexivischer Manier: Die genusdifferenzierenden Endungen -o bzw. - $a$ sind nach Sprecher-Gender verteilt:

(5) MASK: obrigado

(6) FEM: obrigada

Interessanterweise lässt sich allerdings heutzutage in Portugal - zumal in öffentlichen, aber dennoch kolloquialen Kontexten - beobachten, dass Sprecher*innen manchmal diejenige Form verwenden, die eigentlich genau nicht ihrer Gender-Identität entspricht. Der Grund für dieses Phänomen mag in der sehr starken Tendenz zur Nebensilbenabschwächung im Europäischen Portugiesisch zu finden sein. Durch die häufige Realisierung des Endvokals als Schwa scheinen Sprecher*innen deshalb unsicher über die, richtige ' Form zu sein; sie wählen dann manchmal die falsche, wenn sie höflich sein wollen und unreduziert artikulieren. Informellen Beobachtungen zufolge scheinen Frauen diese Vertauschung häufiger vorzunehmen als Männer, so dass eine Neigung besteht, die maskuline Form zu generalisieren, was den üblichen Tendenzen zur Formenneutralisierung in Richtung auf den unmarkierten Wert entsprechen würde. Anders formuliert: Wir beobachten hier eine möglicherweise gerade in Auflösung befindliche Formenopposition, bei der Prozesse phonologischer Reduktion in Kombination mit höflichem Verhalten einen Erklär-Faktor liefern.

Mit den beschriebenen quasi-obligatorischen Routineformeln projizieren Sprecher*innen also mehr oder weniger regelmäßig Sprecher- bzw. Adressaten-Gender, und zwar in stark adressatenorientierten Sprechakten (Gruß, Dank) unabhängig von einer konkret Sprecher/Adressatendeiktischen Formulierung.

Wenden wir uns nunmehr Formen zu, die direkt und zentral Sprechaktrollendeixis kodieren: Personalpronomina mit der Spezifikation 1. bzw. 2. Person.

\footnotetext{
${ }^{7}$ Dies sind nur die häufigsten Partikeln; für weitere Allomorphe und Hinweise zu ihrer Verwendung s. a. a. O., $179-185$.
} 


\section{Genus bei Personalpronomina der 1. und 2. Person}

Hier ist zunächst einmal festzuhalten, dass Genus, wie bereits angedeutet, im Wesentlichen eine Kategorie nominaler Ausdrücke ist. Im Bereich der Personalpronomina ist sie nun nach Ausweis typologischer Untersuchungen ungleichmäßig über die Person-Kategorie verteilt: Dass die Genus-Differenzierung dabei wohl in den Pronomina der 1. Person am seltensten ist, ist schon lange bekannt: „If a language has gender distinctions in the first person, it always has gender distinctions in the second or third person, or in both" (Greenberg 1963: 76). ${ }^{8}$

Wendet man sich den Pronomina der 2. Person zu, also den Anredepronomina, so ist über die dortige Verteilung von Genus-Oppositionen in den Sprachen der Welt m. W. wenig bekannt. Die Karte in Abbildung 1, die aus dem WALS-Material generiert ist, zeigt jedenfalls, dass anredepronominales Genus relativ selten zu sein scheint: Von den insgesamt 378 im Hinblick auf dieses Merkmal kodierten Sprachen besitzen nicht mehr als 20 diese Kategorie. ${ }^{9}$ Interessanterweise sind diese ohnehin schon vergleichsweise wenigen Sprachen auch noch ungleichmäßig verteilt: Eine deutliche Konzentration auf Sprachen in Afrika ist zu erkennen; es handelt sich hierbei sowohl um die weiter unten noch genauer betrachteten afroasiatischen Sprachen als auch um sub-saharische. ${ }^{10}$

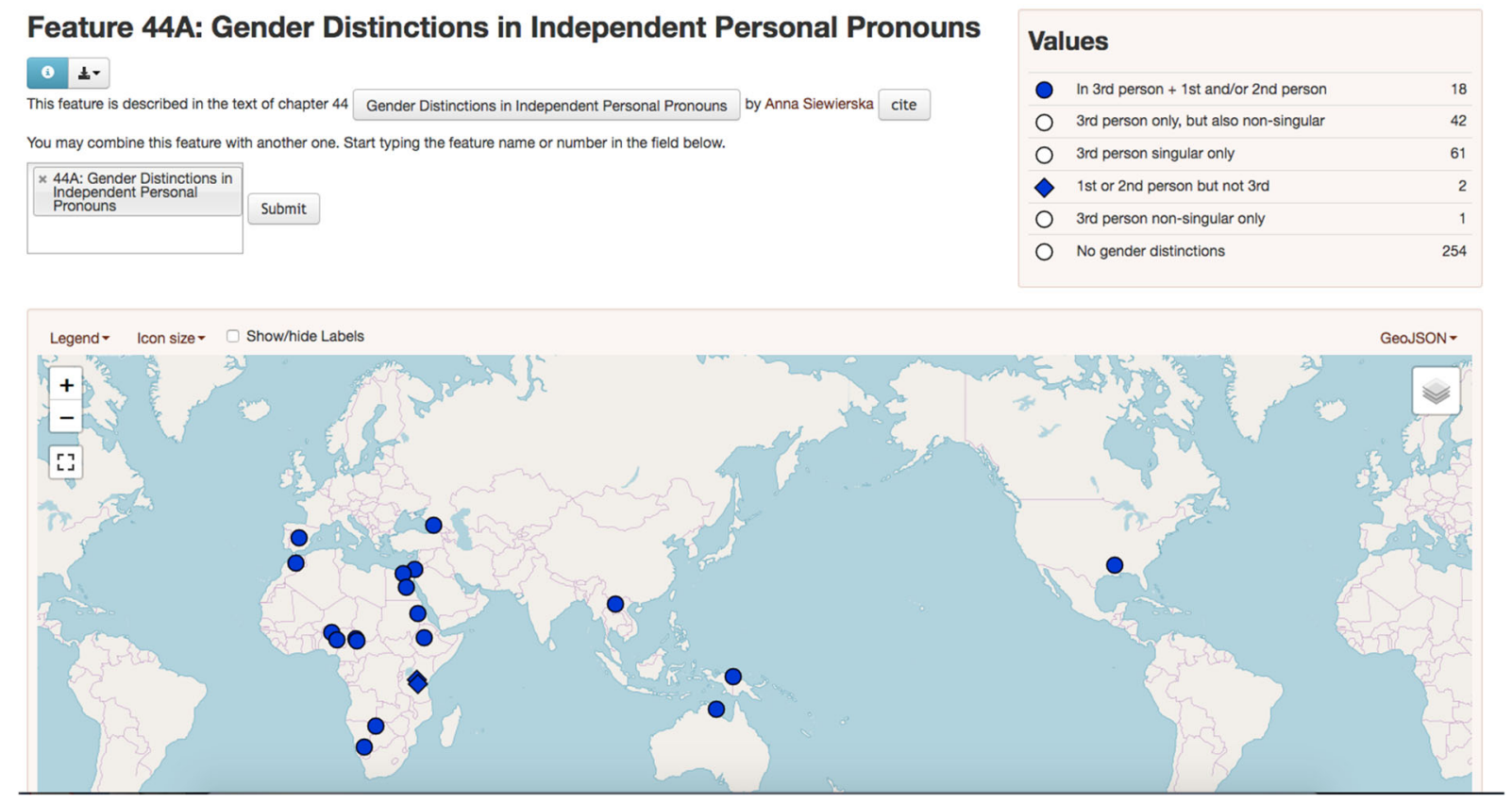

Abbildung 1: Genus in der 1./2.Person laut WALS (Siewierska 2013)

Mit anderen Worten, die in diesem Aufsatz fokussierte Genus-Opposition bei Anredepronomina ist im globalen Kontext eine vergleichsweise seltene Erscheinung, die allerdings eine Reihe von bemerkenswerten Funktionalisierungen aufweist.

\footnotetext{
8 Über die Verhältnisse in der 2. im Vergleich zur 3. Person schreibt Greenberg - soweit ich sehe - nichts.

${ }^{9}$ Aufgrund der Spezifik der Datendarstellung im WALS ist leider nicht ersichtlich, welche der angezeigten Sprachen Genus in der 1., der 2. oder in beiden Personen besitzt. Anrede-Genus kommt also auf jeden Fall nur in einer Untermenge der blau markierten Sprachen in Abbildung 1 vor.

${ }^{10}$ Bhat (2004: 109f.) stellt eine vergleichbare Liste an Sprachen zusammen.
} 


\section{$4 \quad$ Sprecher-Gender in der Anrede - Beispiel Japanisch}

Üblicherweise nimmt die Genus-Kategorie, wenn sie denn überhaupt semantisch basiert ist, auf Eigenschaften des jeweiligen Referenten Bezug, also zum Beispiel dessen Gender oder Belebtheitsgrad oder Ähnliches (cf. Corbett 1991: 7-32). So gesehen mag es vielleicht etwas überraschend anmuten, dass in manchen Sprachen bei den Anredepronomina die Geschlechtsidentität nicht des Adressaten, sondern des Sprechers eine zentrale Rolle spielt. So wird beispielsweise die Verteilung der pronominalen Formen im Japanischen in der weitverbreiteten Darstellung von Shibatani (1990: 371) folgendermaßen visualisiert (Abbildung 2):

\begin{tabular}{|c|c|c|c|c|c|}
\hline \multicolumn{6}{|l|}{$1 \mathrm{st}$ ps. } \\
\hline male speaker & watakusi & watasi & & boku & ore \\
\hline female speaker & watakusi & & watasi & & atasi \\
\hline \multicolumn{6}{|l|}{ 2nd ps. } \\
\hline male speaker & anata & & & kimi anta & omae \\
\hline female speaker & anata & & & & anta \\
\hline \multicolumn{6}{|l|}{ 3rd ps. } \\
\hline & & kare ,he & & & \\
\hline & & $\begin{array}{l}\text { kanozyo } \\
\text {,she' }\end{array}$ & & & \\
\hline
\end{tabular}

Abbildung 2: Personalpronomina im Japanischen (aus Shibatani 1990: 371)

Demnach steht mit anata in formellen Registern nur eine einzige Anrede-Form sowohl für männliche als auch für weibliche Sprecher*innen zur Verfügung (cf. Yonezawa im Druck für eine eingehende Untersuchung dieses Pronomens im Feld des japanischen Anredesystems). Am informellen Pol des Register-Spektrums tritt hingegen eine Differenzierung zutage: Männliche und weibliche Personen verwenden nicht nur für sich selbst, sondern auch für ihren jeweiligen Adressaten unterschiedliche Formen, d. h. omae ${ }_{\mathrm{MASK}}$ bzW. ant $_{\mathrm{FEM}}$ fungieren als sprechergenderspezifizierte Anredepronomina. ${ }^{11}$

Interessant ist nun aber folgende Beobachtung: In der japanischen Gesellschaft sind - wie in sehr vielen Gesellschaften - die Gender-Rollen bis zu einem gewissen Grade aushandelbar, wodurch sich ja gerade ihr Sexus-unabhängiges Wesen manifestiert. Dadurch werden die erwähnten indexikalischen Anredepronomina gewissermaßen frei, um spezifische konversationelle Ausdruckswerte anzunehmen. So beschreibt Abe ([2004]2006: 138f.) die Verwendung von omee, das ansonsten als ,, a very casual 'masculine' second-person pronoun“ fungiert, ${ }^{12}$ in gewissen Situationen „to express [...] extreme rage“, denn ,omee has a certain forcefulness that cannot be expressed by anta or omae, a term that is stereotypically associated with male speakers“. Die Feminin-Form anta wird hingegen benutzt, wenn die sprechende Person einen ,attempt at persuasion" unternimmt.

\footnotetext{
11 Shibamoto Smith (2003: 210) bestätigt dieses Bild, fügt aber weitere Maskulin-Formen am informellen Pol hinzu.

12 Es handelt sich dabei um eine phonologisch reduzierte Variante des informellen omae.
} 
Wie weit verbreitet diese Praxis ist, lässt sich schwer abschätzen, immer wieder ist sie jedoch in queeren Räumen und Gruppen zu beobachten. Abes Daten stammen aus Gesprächsbeobachtungen in lesbisch geprägten Nachtbars in Tokio. Im Deutschen findet sich Ähnliches auch im Nominalbereich, so zum Beispiel die seit den 1920er Jahren etablierte (und bis mindestens in die 1980er fortgeführte) Bezeichnung Kesser Vater für lesbische Frauen, die heute eher als Butch bezeichnet werden. ${ }^{13}$

Nach der gerade dargestellten Interpretation können die japanischen genusdifferenzierten Anredepronomina also im Bereich des Interaktionsmanagements refunktionalisiert werden. So gesehen wird eine als genuin grammatisch zu charakterisierende Opposition pragmatisch umgedeutet: Ihre Funktion ist es dabei, spezielle Sprechakt-Nuancen auf der Ebene des Äußerungsakts zu ermöglichen. - Es wäre aber vielleicht auch möglich, eine ganz andere Deutung des beobachteten Sachverhalts vorzunehmen: Die in Abbildung 2 zitierte Tabelle stammt aus einem für Typolog*innen bestimmten grammatischen Überblickswerk, in dem naturgemäß die systematischen Oppositionen betont werden bzw. in dem die in der Sprache vorhandenen Formvarianten als grammatische Varianten verstanden werden. Eine solchermaßen strukturbezogene Herangehensweise geht jedoch womöglich am Kern der Sache vorbei: Projiziert man nämlich den Kontrast omae-anta nicht auf die Gender-Opposition, dann stellt sich die Sache folgendermaßen dar: Das Formenpaar omae-anta manifestiert zwei Ausdrucksvarianten des Pronomens der 2. Person Singular, deren unterschiedliche Verwendungsbedingungen im Bereich allgemeiner Verhaltensnormen zu suchen sind: Während die eine Form mit ,Nachdrücklichkeit‘, ,kraftvollem Auftreten' oder Ähnlichem assoziiert ist, konnotiert die andere Form ,Bescheidenheit', ,Zurückhaltung' usw. Angesichts des in der japanischen Gesellschaft stark ausgeprägten Drucks nach Sozialverhalten, das mit den traditionellen Gender-Stereotypen konform geht, wird die eine Form im Wesentlichen von männlichen Personen verwendet, die andere hingegen praktisch durchgängig von weiblichen. In dieser Vorstellung sattelt die wahrgenommene Geschlechtsdifferenz bei den Pronomina sozusagen auf einer nicht primär geschlechtsrelevanten Variation auf: Dies ermöglicht es Personen in Queer-Umgebungen, erwünschte Geschlechtszugehörigkeiten mittels des verwendeten Anredepronomens zu markieren, und sei es nur lokal in einzelnen konversationellen Situationen.

Wenden wir uns nunmehr Sprachen zu, bei denen die Genus-Opposition in das Paradigma der Anredepronomina eingebaut ist und auch direkt Bezug nimmt auf das Gender der angeredeten Person.

\section{Adressaten-Gender in der Anrede - Beispiel Arabisch und andere semitische Spra- chen}

Am prominentesten ist Anrede-Genus sicherlich in den afroasiatischen Sprachen, und zwar sowohl in deren semitischem Zweig als auch in den Berber-Sprachen. Zunächst soll hier exemp-

\footnotetext{
13 Anekdotisch sei zudem auf die während einiger Jahre in der Bar „Die Kleine Philharmonie“ in Berlin-Wilmersdorf zu beobachtende diskursive Praxis verwiesen, bei der die Stammgäste habituell - und augenzwinkernderweise - auf einen jungen männlichen Angestellten mit der Bezeichnung die Praktikantin referierten; die zugehörige Pronominalform war meist sie. - Für das Britische Englisch ist eine ähnliche, dort habitualisierte Praxis unter dem Begriff Polari beschrieben in Baker (2019: 92-94).
}

ISSN 1615-3014 
larisch das Arabische in einigen seiner gegenwärtigen Ausprägungen betrachtet werden. Hinsichtlich ihrer Struktur entsprechen die Paradigmen der Personalpronomina der modernen Varietäten des Arabischen allesamt dem des Hoch-Arabischen - wenn auch mit jeweils spezifischen phonologischen Formen gefüllt:

\begin{tabular}{|l|l|l|l|}
\hline & SG & DU & PL \\
\hline 1 & 'anaa & & naHn-u \\
\hline 2 MASK & 'anta & 'antumaa & 'antum \\
\hline 2 FEM & 'anti & & 'antunna \\
\hline 3 MASK & huwa & humaa & hum \\
\hline 3 FEM & hiya & & hunna \\
\hline
\end{tabular}

Abbildung 3: Personalpronomina im Hoch-Arabischen (nach Ryding 2005: 298f.)

Während die Pronomina im Dual lediglich eine genusneutrale Form besitzen (die offenbar von der Plural-Maskulin-Form abgeleitet ist), differenzieren die 2. und die 3. Person sowohl im Singular als auch im Plural nach Genus. In den weitaus meisten Fällen werden diese Pronomina auch genau in der erwartbaren Weise verwendet, also anta und antum als Anrede an (eine) männliche Person(en) bzw. anti und antunna als Äquivalente für (eine) weibliche Person(en); für gemischtgeschlechtliche Gruppen gilt die Maskulin-Form, auch dies wie erwartbar.

Interessant ist nun, dass bei gewissen Personen bzw. in gewissen Registern oder auch nur in gewissen Situationen manchmal Abweichungen von diesen Regularitäten zu beobachten sind. So ist beispielsweise im Golf-Arabischen von Abu Dhabi die Distinktion im Plural in Auflösung begriffen, und zwar dahingehend dass - ähnlich wie im Dual - die offenbar als unmarkiert geltenden Maskulin-Formen generalisiert werden (cf. Qafisheh 1977: 159). - In anderen Fällen scheint es genau umgekehrt zu sein: So werden im Tunesischen Arabischen die Maskulin-Feminin-Formen von manchen Sprecher*innen nachgerade ,austauschbar' verwendet. Insbesondere die Form inti2sG.Fem wird dabei genusneutralisiert eingesetzt. Eine solche Verwendung nimmt dabei aber häufig soziale Bedeutung an, und zwar insofern als sie nachgerade indexikalisch für Urbanität wirkt: Menschen, die solchermaßen neutralisieren, markieren dadurch ihren Status als ,urbanite and Francophile Tunisians“; für die ländliche Bevölkerungsgruppen wirkt sie somit als Zeichen einer ,new identity that they have acquired through education and hard work“" (Eirini Theodoropoulou, pers. Mitt.).

Neben dieser sozialindexikalischen Funktionalisierung, die gewissermaßen auf der MakroEbene der Identitätskonstruktion operiert, sind in mehreren Varietäten des Arabischen Praktiken zu beobachten, bei denen konventionalisierterweise diejenige Anredeform benutzt wird, die angesichts des angenommenen Genders des Adressaten die falsche ist. Beispielsweise ist es im Libanon in sehr informellen Situationen während eines Streits möglich, gegenüber einem Mann die Feminin-Form inti zu gebrauchen. Hier dient die Pronomenwahl also ganz konkret als verbales Aggressionsmittel: Das feminin-spezifizierte Anredepronomen bewirkt eine direkte symbolische Entmännlichung des Adressaten. - Im Jemen wiederum kann das PluralPronomen als höfliche Anrede auch an einzelne Person verwendet werden; ${ }^{14}$ dies betrifft im

\footnotetext{
${ }^{14}$ Dies ist insofern bemerkenswert als das Arabische - im Gegensatz zu vielen anderen Sprachen - Höflichkeit üblicherweise nicht pronominal markiert, sondern stattdessen auf eine ganze Palette nominaler Formen zurückgreift.
} 
Wesentlichen die ehrende Anrede an ,alte Leute'. Will man nun heutzutage ${ }^{15}$ einer alten Frau besondere Ehre zuteil werden lassen, so verwendet man dafür die Plural-Maskulin-Form intum. Hier wirkt die Zuschreibung des Männlichkeitsmerkmals also als konversationell ehrend.

Diese Beobachtungen mögen einen Eindruck von der Vielfalt der Möglichkeiten geben, die das Arabische bereitstellt, um vermittels der Manipulation der Opposition im Anrede-Genus zusätzliche Bedeutungskomponenten zu generieren. Diese pragmatische Zusatzschicht, die gewissermaßen über der grammatischen Distinktion liegt, ist in allen Varietäten des Arabischen vorhanden; sie kann jeweils varietär unterschiedlich konventionalisiert sein und dadurch konversationell-lokal unterschiedlich eingesetzt werden.

Eine solche Refunktionalisierung von Anrede-Genus ist auch in einer Reihe anderer semitischer Sprachen zu finden. So wird das Anrede-Genus im Modernen Hebräischen laut Tobin (2001) in diesem Sinne variabel eingesetzt: Er erkennt in der Bezugnahme auf weibliche Personen mittels maskuliner Formen einen Beigeschmack von Affektivität und allgemeiner Gemeinsamkeitsbekundung im Hinblick auf die Sprecher-Adressat-Relation. ${ }^{16}$ - Genau dieselben Bedeutungskomponenten ,rapprochement - endearment - closeness“ werden von Wołk (2009: 131) als „most common“ im Amharischen bezeichnet; Wołk beschreibt aber auch die beiden oben für das Arabische erwähnten Funktionen: Maskulin als Lob für Frauen, unabhängig vom Alter der Angeredeten, und Feminin als Beleidigung für Männer. - Teferra/Beyl (1978/79: 67) erwähnen sogar, dass solche Beleidigungen im Tigrinya bereits als Auslöser von Gerichtsverfahren gewirkt haben. ${ }^{17}$

Es lässt sich also zusammenfassen, dass in allen betrachteten semitischen Sprachen Vertauschungen auf der Ebene der Genus-Gender-Dichotomie zu verzeichnen sind. ${ }^{18}$ Die damit einhergehenden pragmatischen Funktionen sind einander sehr ähnlich: Die Hauptfunktion scheint im Bereich der Status-Übertragung zu liegen: Als weiblich konzeptualisierten Personen werden

\footnotetext{
15 Diese Einschränkung bezieht sich darauf, dass einer meiner Informanten, der bereits in den 1980er Jahren aus dem Jemen ausgewandert ist, eine solche Praxis als vollkommen unmöglich ablehnt.

${ }^{16}$ Interessanterweise scheint es im Bibelhebräischen genau umgekehrt gewesen zu sein: Hier konnten femininmarkierte Formen in der Anrede an männliche Personen verwendet werden (Muchnik 2016: 28), wobei die Funktion dessen unklar ist.

17 Von den eben beschriebenen Verwendungsweisen zu unterscheiden sind diejenigen, die Sa'ar (2007: 412ff.) wiederum für das Moderne Hebräisch beschreibt: Was dort als ,pseudo second person“ bezeichnet wird - eine gängige andere Bezeichnung wäre ,indefinit-generisches Pronomen “-, ist der Gebrauch von nur einem der beiden im Prinzip zur Verfügung stehenden Anredepronomina bei verallgemeinernden Formulierungen. Insofern es sich hier gar nicht um eine Anrede im engeren Sinne handelt, gehört dieses Phänomen nicht zu den in vorliegendem Aufsatz behandelten. Es verwundert aber jedenfalls nicht, dass dabei die grammatisch unmarkierte, eben maskuline Form genommen wird. Denn dies ist strukturell vergleichbar mit der generellen Verwendung des im Hinblick auf Respekt unmarkierten $d u$ in indefinit-generischen Kontexten im Deutschen, und zwar auch gegenüber Personen, die eigentlich gesiezt werden (cf. dazu Simon 2003: 161).

18 Über die soziopragmatischen Verhältnisse in den strukturell vergleichbaren Berber-Sprachen sind mir keine relevanten Fakten bekannt; die von mir konsultierten Grammatiken und Überblicksdarstellungen erwähnen nichts Einschlägiges.
} 
lobenderweise männliche Eigenschaften zugesprochen bzw., umgekehrt, männlichen Personen wird ihre Männlichkeit abgesprochen, wodurch ein starker Beleidigungseffekt eintritt. ${ }^{19}$

\section{Adressaten-Gender in der Anrede - Beispiel Älteres Deutsch (und Romania)}

Neben den bislang besprochenen Sprachen, deren Wahl sich aus den $W A L S$-Daten ergeben hat, sind aber auch weitere Sprachen relevant, die - soweit ich sehe - bislang noch an keiner Stelle in die allgemeine Diskussion zum Anrede-Genus Eingang gefunden haben (und zwar weder im Bereich der typologischen Forschung zu den Personalpronomina noch in der Forschung zur grammatischen Kategorie Genus, und auch nicht in der Forschung zu Sprache und Gender). Das einschlägige Phänomen als solches ist in der historisch-philologischen Forschung zum Deutschen hinlänglich bekannt, wird allerdings auch dort bislang nicht in Bezug gesetzt zur hier behandelten Thematik: Es handelt sich dabei um die höfliche Anrede an eine einzelne Person im Deutschen der Frühen Neuzeit.

Bekanntermaßen ist die Geschichte der Anredepronomina des Deutschen eine besonders reichhaltige, die sich in einem jahrhundertelangen Prozess über mehrere Stufen vollzogen hat, wobei die jeweilige grammatisch-kategorielle Spezifikation der involvierten Formen durch ein komplexes Zusammenspiel von Person, Numerus und auch Genus zustande kam (für eine kompakte Darstellung cf. Nübling et al. 2017: 207-212; ausführlicher Simon 2003: 92-133). - Sowohl am Ausgangs- als auch am Endpunkt der Entwicklung (alt-/mittelhochdeutsch $d u$ vs. ir bzw. Gegenwartsdeutsch $d u$ vs. Sie) spielt Genus keine Rolle. In den dazwischenliegenden Phasen jedoch gab es mit $e r_{\mathrm{MASK}}$, si $_{\mathrm{FEM}}$ und $e s_{\mathrm{NEUT}}$ singularische Pronomina in Anredefunktion, die für Genus spezifiziert waren.

Zunächst ist zu bemerken, dass alle drei Pronomina auch wirklich in adressatenbezüglicher Funktion belegt sind. Der Grund dafür liegt in der Tatsache, dass solche Verwendungen von Pronomina der 3. Person zunächst - in der frühneuhochdeutschen Periode - ausschließlich auftauchen, wenn vorher im Diskurskontext bereits eine indirekte Nominalanrede des Typs der Herr oder die Jungfrau verwendet worden ist. Die Pronomina sind hier also als rein anaphorische Formen zu interpretieren, weshalb in solchen Anrede-Konstruktionen auch niemals die deiktischen $d$-Varianten der Pronomina zu finden sind (zu diesen cf. Simon 2003: 49-51). Ihre Genusspezifikation wird dabei determiniert durch das Genus des anaphorisch wiederaufgenommenen Nomens. Da die meisten Personenbezeichnungen im Deutschen dasjenige Genus tragen, das dem angenommenen Gender des Referenz-Individuums entspricht (maskulin-männlich, feminin-weiblich), ist in den meisten Fällen nichts Besonderes zu beobachten. Allerdings gibt es einige wenige ehrende Bezeichnungen für weibliche Personen, die neutral sind, die mithin als genusbezogene Hybridnomina anzusehen sind. Deshalb gibt es Belege folgenden Typs (adressatenreferentielle Formen unterstrichen):

\footnotetext{
${ }^{19}$ Manche der vorstehenden Informationen sind - in anderer Perspektivierung und Gewichtung - auch in das typologische Überblickswerk von Aikhenvald (2016: 99-119) eingegangen. Für eine Perspektivierung von lexikalischen Genuszuweisungsvertauschungen im Hinblick auf Gender-Konzepte in den jeweiligen Kulturen cf. Aikhenvald (2019) und als Ansatzpunkt für eine pragmatische Ableitung von evaluativen Strukturen cf. Steriopolo (2019).
} 
(7) Wan es mein gnädiges Fräulein im bästen vermärken wolte, so könt' ich $\underline{\mathrm{Ihm}}$ noch wohl den wahren sün gnugsam eröfnen.

(Philip von Zesen 1645; zitiert in Keller 1904/05: 172)

Da die er-sie-es-Formen in dieser Periode noch nicht alleine, ohne entsprechendes Antezedens, auftreten können, wäre es falsch, sie als Anredeformen im engeren Sinn zu verstehen; sie gewinnen ihre referentielle Kraft nämlich lediglich indirekt, durch den Rückbezug auf ihr Nomen.

In jener Zeit werden nicht nur schlichte Personenbezeichnungen für nominale Indirektanreden verwendet, sondern es wird ein breites Spektrum von Nominalabstrakta in Anrede-Funktion rekrutiert. Dabei wird gewissermaßen eine besonders hervorstechende positive Eigenschaft des Adressaten angeredet, und dadurch - metonymisch - auch der Adressat selbst; hierher gehören Abstrakta wie Gelehrtheit, Hochwürdigkeit, Majestät, Gnade, Ehre. ${ }^{20}$ Anreden mit solchen Abstraktnomina, die allesamt Feminina sind, führen bei männlichen Adressaten wiederum zu einem Status als Hybridnomen, was sich sehr schön mit folgendem Dialogfragment aus einer Tragödie von Andreas Gryphius illustrieren lässt (adressatenreferentielle Formen unterstrichen):

(8) - (Juxton:)

- (Carol:)

-(Juxton:)

- (Carol:)

- (Juxton:)
Der höchste, wehrter fürst! woll ihn den tag anblicken!

Wir gläuben, dass er werd uns, seinen knecht erquicken.

Drückt ihre majestät noch ein verborgen leid?

Wir finden uns getrost und zu der noth bereit.

Hat sie der kurtzen nacht genossen sonder sorgen?

(Andreas Gryphius ca. 1650; zitiert in Vennemann/Wagener 1970: 20)

Während Carol zu Beginn mit dem Maskulin-Pronomen angeredet wird, zeigt der letzte Satz eine Feminin-Form als anaphorische Wiederaufnahme von Majestät. Anders als in den semitischen Beispielen in Abschnitt 5 oben, ist hier mit dem Genus-Wechsel aber kein pragmatischer Wert verbunden, vielmehr ist er syntaktisch induziert ohne spezielle Bedeutungskomponente. Lediglich die Tatsache der Verwendung eines Pronomens der 3. Person Singular in Anredefunktion löst an sich eine Höflichkeitsinterpretation aus.

Der entscheidende Schritt hin zu einem echten Anrede-Genus erfolgt zu Beginn des 17. Jahrhunderts. ${ }^{21}$ Nunmehr sind - teils in freier (?) Variation zu anaphorischen Verwendungen - Pronomina zu finden, bei denen Genus und Gender konform gehen: An dieser Stelle lässt sich nun sagen, dass das Deutsche mit $e r_{\mathrm{MASK}}$ und sie $_{\text {Fем }}$ eine strenge Genus-Opposition bei den Anredepronomina eingeführt hat. Die Verteilung der beiden Formen ist hinsichtlich dieser GenusOpposition unproblematisch (soweit das aus den Quellen erschließbar ist). Aber natürlich bilden auch sie nur einen Teil des anrederelevanten Formeninventars: Sie stehen in einem soziolinguistisch bedingten Paradigma mit den Pronomina $d u$ und $i h r$, die beide auch in der Anrede

\footnotetext{
${ }^{20}$ Da viele dieser Nominalabstrakta in die Deklinationsklasse der schwachen Feminina gehörten (und dadurch in gewissen Kasus die Numerusunterscheidungen systematisch unklar waren), ist diese Praxis einer der Faktoren, die zur Herausbildung der gegenwärtigen (genusindifferenten) Sie-Anrede geführt haben; Genaueres dazu bei Listen (1999).

${ }^{21}$ Laut Metcalf (1938: 64) lässt er sich auf die Mitte der ersten Jahrhunderthälfte datieren. - Es sei an dieser Stelle angemerkt, dass eine saubere, quellengesättigte Studie zur Entwicklung der hier beschriebenen Anrede-Verhältnisse im 17. und 18. Jahrhundert weiterhin fehlt.
} 
an eine einzelne Person Verwendung finden. $\mathrm{Zu}$ den Faktoren, die die Verteilung der Formen über die Individuen hinweg steuern - aber teils auch ihre Variation innerhalb einzelner Gespräche -, gehören der soziale Stand der Interaktanten, die Art ihrer Sozialbeziehung usw., aber auch gesprächsstrategische Erwägungen (zu letzteren als Faktor bei gesprächsinterner Variation cf. Simon 2016); sie lassen sich insgesamt als die üblichen höflichkeitsrelevanten Parameter zusammenfassen. ${ }^{22}$ Die Genus-Kategorie als solche spielt hier keine Rolle; lediglich ihre Position an der Spitze der dreigliedrigen Höflichkeitshierarchie $d u$-ihr-er/sie ist relevant.

Die deutsche Sprachgeschichte bietet im Hinblick auf die höfliche Pronominalanrede ein weiteres bemerkenswertes Phänomen: Für einen relativ kurzen Zeitraum am Ende des 18. und am Beginn des 19. Jahrhunderts lässt sich beobachten, dass die sozial-pragmatische Wertigkeit der er/sie-Anrede abnimmt und diese Anrede im Höflichkeitswert gewissermaßen unter die Anrede mit $i h r$ absinkt:

Um das jahr 1780 ungefähr behauptete noch das er, sie (sg.) seinen bisherigen rang vor dem ihr. [...] Heutzutage, seit wieder ein halbes jh. abgelaufen ist [ca. 1830; HS], hat sich die lage von $i h r$ und $e r$ verrückt, das $e r$ ist unter das $i h r$ herabgesunken. die in der vorigen periode geerzt wurden, erhalten jetzt plurales sie, die damals geirzten aber er. [...] ihr hat wieder eine edlere geltung, gleichstehende, in höheren ständen, bedienen sich seiner nicht selten.

(Grimm 1898: 369f.)

Die Gründe für diese aus typologischer Sicht erstaunliche Entwicklung - gemäß der Beobachtungen von Head (1978: 191) ist ein solches System, bei dem Numerus-Variation höflicher ist als Person-Variation höchst ungewöhnlich - sind wohl vielfältig: Neben makrosoziolinguistischen Faktoren wie gesellschaftlicher Umbau oder Kontakteinfluss des Französischen spielt womöglich auch die Grammatik direkt eine Rolle. Ein Grund mag nämlich gerade in der Tatsache der Genus-Spezifikation als solcher liegen: semantisch/grammatisch stärker spezifizierte Formen (hier: er/sie) eignen sich generell weniger als Höflichkeitsformen als weniger spezifizierte (hier: ihr, cf. Simon 2003: 120-124 für eine typologisch informierte Diskussion).

Die deutsche Standardsprache der Gegenwart besitzt nun mit der $d u$-Sie-Opposition keine genusrelevante Differenzierung mehr; dennoch gibt es wohl auch heute noch - vereinzelt - nach Adressatengender variierende Anredepronomina (solche mit der Spezifikation ,3. Person Singular Maskulin bzw. Feminin'), und zwar als sehr seltene konversationelle Option in Norddeutschland. ${ }^{23}$

Das Deutsche ist im übrigen nicht die einzige europäische Sprache, die in der Frühen Neuzeit einen Entwicklungsschub im Bereich der Anredepronomina macht. Die oben beschriebene Praxis der indirekten Nominalanreden - auch mit Abstrakta - war weit verbreitet in den germani-

\footnotetext{
${ }^{22}$ Auch in dieser Hinsicht steht eine genauere, modernen historisch-soziolinguistischen und pragmatischen Methoden verpflichtete Untersuchung noch aus.

23 Die Datenlage hierzu ist allerdings sehr dürftig. - Voraussetzung scheint jedenfalls zu sein, dass es sich um ein (ehemals) niederdeutsches Dialektgebiet handelt (im Niederdeutschen war in der ersten Hälfte des 20. Jahrhunderts die Anrede mit der 3. Person Singular noch weit verbreitet). Die Hauptfunktion scheint - neben einer gewissen Scherzhaftigkeit - die Vermeidung der Entscheidung zwischen $d u$ und Sie zu sein, die beide in gewissen Situationen als unangemessen gelten können (cf. Simon 2003: 126f. für ein paar diesbezügliche Beobachtungen).
} 
schen und den romanischen Sprachen (cf. Hummel 2020 für einen aktuellen Forschungsüberblick zum Spanischen und Portugiesischen, v. a. 39f.). ${ }^{24}$ Dies hat bis heute Auswirkungen auf die jeweiligen Anredesysteme. So stellt beispielsweise das gegenwärtige spanische Höflichkeitspronomen usted eine phonologisch reduzierte und univerbierte Form von vuestra merced ,Euer Gnaden“ dar, und portugiesisch você geht auf vossa mercê zurück. Hier ist also offenbar der Wortkörper einer besonders prominenten possessiven Nominalabstraktkonstruktion selbst tradiert worden. - Anders verhält es sich mit dem Italienischen, dessen Entwicklung eher dem Deutschen vergleichbar ist: Als höfliches Anredepronomen fungiert dort heute Lei, welches gleichzeitig die nicht-nominativische Form des Pronomens 3. Person Singular Feminin ist. Im gegenwärtigen Standarditalienischen wird diese Form unterschiedslos für Personen jedweden Geschlechts verwendet. Ihr Ursprung liegt natürlich in der Tatsache begründet, dass auch im Italienischen die ehrenden Abstrakta zur Klasse der Feminina gehören, dass mithin in ihrer anaphorischen Wiederaufnahme stets das Feminin-Pronomen verwendet wurde. ${ }^{25}$ - Dennoch ist auch im Gegenwartsitalienischen eine echte Genusunterscheidung bei den Anredepronomina vorhanden, denn die Generalisierung des Femininums über alle Personen hinweg ist im Wesentlichen ein Phänomen der Standardsprache: Während süditalienische Varietäten entweder nie über die 2. Person Plural hinausgegangen und beim voi geblieben sind oder - ähnlich dem Iberoromanischen - eine Possessivkonstruktion phonologisch zu vossía und seinen Varianten reduziert haben -, haben viele norditalienische Dialekte eine Genus-Opposition in der 2. Person innoviert (cf. Renzi 1996 und Ledgeway 2015: 93-96 für Zusammenstellungen der dialektalen Verhältnisse). ${ }^{26}$ Im Unterschied zum Deutschen des frühen 17. Jahrhunderts scheint es in diesen italienischen Dialekten aber so zu sein, dass die alte Pluralform nicht mehr als Höflichkeitsform Verwendung findet; d. h. das System kennt weiterhin nur zwei Hierachiestufen auf der Höflichkeitsskala, nur halt mit der Besonderheit, dass der höfliche Pol zweigeteilt ist je nach Adressaten-Gender. ${ }^{27}$

Zusammenfassend lässt sich zum Deutschen und zu den romanischen Sprachen Folgendes festhalten: Die weitverbreitete Praxis der sogenannten, indirekten Anrede' mit nominalen Ausdrücken - sowohl mit Personenbezeichnungen als auch mit Abstrakta in Possessivkonstruktionen - führte zur Verwendung pronominaler Formen in Anredefunktion, die ,eigentlich ' in anaphorischer Funktion eingesetzte Formen der 3. Person sind (kenntlich an der bei Verwendung als

\footnotetext{
${ }^{24}$ Für das dem Deutschen vergleichbare frühneuzeitliche Schwedisch möge dieses Beispiel aus Svennung (1958: 111) genügen; es stammt aus der Predigt anlässlich der Krönung König Gustavs I Wasa im Jahre 1528, gerichtet

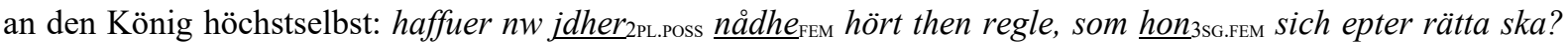
(wörtl.: ,Haben nun Euer Gnaden gehört die Regel, nach der sie sich richten muss?').

25 Übrigens erschien es manchem in der Zeit des Faschismus als unitalienisch und unmännlich, mit einem höflichen Anredepronomen im Feminin angeredet zu werden; dementsprechend gab es eine (wenig erfolgreiche) Kampagne zur Generalisierung der süditalienischen Höflichkeitsanrede mit genusindifferentem Plural-voi (cf. Cicognani 1938).

${ }^{26}$ Zur Geschichte der italienischen Höflichkeitsanrede cf. Grand (1930); hier S. 86 auch Erwähnung der GenusOpposition.

27 An dieser Stelle soll der Hinweis genügen, dass auch das Afrikaans adressatengenderspezifische Höflichkeitspronomina besitzt: Viele der höflichen Pendants zum unmarkierten $j y$ sind geschlechtsbestimmt, z. B. oom (abgeleitet vom Wort für ,Onkel') oder tannie (,Tante'). Für Näheres zur Grammatik der afrikaansen Anredepronomina cf. Simon (2010).
} 
Subjekt kongruierenden Verbalform). Diese refunktionalisierten Pronomina tragen dann als Ergebnis ihrer Entstehungsgeschichte - quasi als Nebeneffekt - Genusmarkierung; im Deutschen aufgrund seines dreigliedrigen Genussystems anfangs manchmal sogar auch mit Neutrum-Spezifikation. Im Deutschen des 17. Jahrhunderts und in heutigen nördlichen Dialekten des Italienischen lösen sich dann diese genusspezifizierten Anredepronomina aus ihrer anaphorischen Klammer und werden selbständig verwendet - die Genus-Spezifikation ausgerichtet am Gender des Adressaten. Hinweise auf Genus-Gender-Reibungen wie in Abschnitt 5 für die semitischen Sprachen beschrieben sind mir weder für das Deutsche noch für das Italienische bekannt.

\section{Zusammenfassung: Grammatik und Pragmatik von Genus im Adressatenbezug - sowie: Methodologische Reflexion}

Die vorliegende, ganz sicher nicht erschöpfende Umschau in den Sprachen der Welt hat eine Reihe von Phänomenen fokussiert, bei denen die soziale Kategorie Gender bei der Verwendung von sprachlichen Formen eine prominente Rolle spielt, häufig vermittelt über die grammatische Kategorie Genus. Einige der exemplarischen Beobachtungen seien hier stichpunktartig wiederholt:

- Im Französischen und im Portugiesischen wird Adressaten-Gender bei höflichkeitsbezogenen Routineformeln relevant.

- Pronominale Systeme - insbesondere solche mit vielgestaltigen Paradigmen, beispielhaft wurde das Japanische herausgegriffen - erlauben die Appropriation gewisser Pronomina im Dienste der Konstruktion der Gender-Fluidität der Sprecher*innen.

- In verschiedenen Kulturen werden genusspezifizierte Formen für das höflichkeitssensitive Interaktionsmanagement eingesetzt: Im Japanischen verleihen maskulin-spezifizierte Formen dem Sprecher - genauer: der Sprecherin - Autorität. In Varietäten des Arabischen (und in anderen afroasiatischen Sprachen) können die genusspezifizierten Anredeformen strategisch genutzt werden: So führt die symbolische Kastration vermittels der FemininAnrede an eine männliche Person zu einem beleidigenden Effekt, und umgekehrt kann eine (ältere) Frau geehrt werden, indem man sie durch Maskulin-Anrede kurzfristig zu einem Ehren-Mann ernennt.

- In der Geschichte des Deutschen und des Schwedischen führten höfliche indirekte Nominalanreden zur Verwendung anaphorischer Pronomina, die im Falle von GenusHybridnomina auch selbst Höflichkeit zum Ausdruck brachten, qua Genus-GenderReibung. Durch Genus-Gender-Parallelisierung wird in einem weiteren Schritt im Deutschen - genauso wie in norditalienischen Dialekten - der Höflichkeitspol der Anredepronomina gespalten: Die Pronomina werden adressatengender-adäquat verwendet; Spielereien des semitischen Typs scheinen nicht vorzukommen.

Eine Studie wie die vorliegende kann nicht mehr bieten als einen ersten, notgedrungenermaßen oberflächlichen Eindruck von der Vielgestaltigkeit des Phänomens: Anrede-Genus kommt in ganz unterschiedlichen grammatisch-pragmatischen Kontexten in ganz unterschiedlichen Sprachen vor. - Eine solche Skizze wirft sogleich eine Reihe von methodologisch und theoretisch relevanten Fragen auf; sie bietet Anlass zur Reflexion allgemein-linguistisch inspirierten Arbeitens. 
Die hier untersuchten Fakten konnten beigebracht werden auf Grundlage der sprachbeschreibenden Arbeit vieler Kolleg*innen; eigene Erhebungen erfolgten nur punktuell und eher anekdotisch zum Deutschen und zum Arabischen. Die vorliegende Umschau beschränkt sich also im Wesentlichen auf die Auswertung wissenschaftlicher Literatur, die bei seltener betrachteten Sprachen häufig genug nur in Form von deskriptiven Grammatiken vorliegt. Nun liegt es in der Natur der Sache, dass grammatische Beschreibungen ihr Hauptaugenmerk auf sprachsystematische Aspekte legen - Phänomene wie das hier interessierende, die auf der Ebene der soziolinguistisch gekoppelten Pragmatik (Höflichkeit) und teilweise sogar der Gesprächsführung angesiedelt sind, fallen dabei häufig unter den Tisch. Aus der Tatsache, dass bei gewissen Sprachen zwar das Vorhandensein von im Hinblick auf Genus differierende grammatische Varianten konstatiert wird, dann aber keine weitere Informationen zu Genus-Gender-Reibungen geliefert werden, darf keineswegs geschlossen werden, eine solche Funktionalisierung sei in der jeweiligen Sprache unüblich. Oftmals ist zu vermuten, dass die Beschreibung an dieser Stelle nicht detailliert genug ist bzw. dass der*die Autor*in das Phänomen als nicht-grammatikrelevant aus der Darstellung ausgeschieden hat. Selbst bei soziolinguistischen Detailstudien ist in dieser Hinsicht Vorsicht geboten; so erwähnt beispielsweise die an sich sehr detailreiche und reflektierte Studie von Braun (1984) keinerlei Variabilität der Anrede-Genus-Verwendung im Jordanischen Arabischen. ${ }^{28}$ Insofern Grammatiken und andere nicht auf unser spezifisches Phänomen fokussierte Studien also eine problematische Quelle sind, wäre es höchst wünschenswert, eine größere, umfragegestützte, systematisch vergleichende Untersuchung anzugehen, was gerade in den afroasiatischen Sprachen zu interessanten Erkenntnissen über den Zusammenhang von durch grammatische Strukturbildung zur Verfügung stehendem Formeninventar einerseits und den Gender-Konzeptionen in den jeweiligen Kulturen andererseits führen sollte. - Generell ist in diesem Zusammenhang zu fragen, inwieweit es überhaupt sachlich angemessen ist, die grammatischen Formen, von denen es bei Anrede-Genus typischerweise zwei gibt, auf die tradierte Sexus-Dichotomie zu projizieren. Zumindest der skizzierte Fall des Japanischen sollte hier vorsichtig stimmen, denn nicht immer geht die Gender-Skala parallel zur Formenopposition. Die traditionell-binaristisch argumentierenden wissenschaftlichen Darstellungen reifizieren also womöglich ein Phänomen, das als solches in der gelebten sprachlichen Wirklichkeit gar nicht vorkommt.

In diesem Kontext ist es auffällig, dass der beschriebene Refunktionalisierungsmechanismus innerhalb der afroasiatischen Sprachfamilie weit verbreitet ist - wenn auch mit je unterschiedlicher inhaltlicher Füllung, was die (Un)Höflichkeitsinterpretation anbetrifft. Es stellt sich die Frage, ob die Verbreitung über sehr viele Sprachen in der Sprachfamilie und über einen relativ großen geographischen Raum hinweg allein durch die grammatische Struktur gestützt ist - immerhin geben die Paradigmen die Genus-Differenzierung einfach her, auch ohne die Höflichkeitsdimension wie im Deutschen und im Italienischen -, oder ob als weitere Faktoren die soziokulturellen Gegebenheiten wie beispielsweise die Konzeptualisierungen von Gender in den jeweiligen Gesellschaften eine Rolle spielen. Augenfällig sind zumindest die Auf- bzw. Abwertungsprozesse, die mit der Genus-Gender-Vertauschung einhergehen. Denn bekanntlich sind die soziopragmatischen Verwendungsbedingungen sprachlicher Formen ein besonders

\footnotetext{
28 Vielleicht ist aber auch das Jordanische Arabische wirklich speziell, indem es - anders als die anderen Varietäten - eben keine Höflichkeitsfunktionalisierung von Anrede-Genus zulässt.
} 
volatiler Bereich; sie unterliegen vergleichsweise kurzfristigem Wandel. ${ }^{29}$ Auch aus diesem Grunde wäre eine gesonderte Untersuchung der genannten Sprachen lohnenswert.

Bisher noch gar nicht zur Sprache gekommen ist die Tatsache, dass die Anredepronomina natürlich nicht im luftleeren Raum existieren. Sie sind vielmehr via Kongruenz in komplexe grammatische Beziehungsgeflechte eingebunden: Sowohl in den afroasiatischen als auch in den romanischen Sprachen kongruieren verbale Formen (Partizipien) und nominale Formen (Adjektive) in bestimmten Konstellation nach Anrede-Genus. Bezüglich der Verhältnisse in HybridVerwendungen ist den bisherigen grammatischen Darstellungen keine Information zu entnehmen. Also: Wie kongruieren Adjektive/Partizipien, wenn eine männliche Person mit dem femininen Anredepronomen angeredet wird? Auch hier wäre nachzubohren. ${ }^{30}$

Was lässt sich also nun insgesamt lernen aus der Betrachtung der grammatischen Genus-Opposition, wie sie uns in Anrede-Konstellationen in verschiedenen Sprachen entgegentritt? Zunächst ist auf der Form-Ebene festzuhalten, dass das Phänomen weltweit gesehen eher selten ist und sich zudem in gewissen Sprachfamilien/Regionen bündelt. Allerdings konnten durch den Einbezug historischer Sprachstufen des Deutschen sowie dialektaler Varietäten des heutigen Italienischen neue einschlägige Beispiele beigebracht werden. Während manche der Systeme relativ rigide funktionieren, da sie über grammatische Kongruenzrelationen geregelt sind (es sind diejenigen, bei denen Höflichkeitskomponenten direkt in die Semantik der Formen eingebaut sind), sind andere freier organisiert; hier sattelt (Un)Höflichkeit auf einer reinen Genus-Opposition auf. Anders formuliert: Verschiebungen entlang der Genus-Distinktion führen via eine damit einhergehende Gender-Interpretation zu pragmatischen Effekten auf der Ebene der Beziehungsorganisation. An dieser Stelle offenbart sich der Bezug zu den Femineutra und zu anderen Arbeiten in diesem Band: Genus ist womöglich eine weniger stark festgelegte grammatische Kategorie als traditionellerweise angenommen; Genus ist stattdessen wohl - in begrenztem Maße - pragmatisch aufladbar.

\section{Literaturverzeichnis}

Abe, Hideko ([2004]2006): „Lesbian bar talk in Shinjuku, Tokyo“. In: Cameron, Deborah/Kulick, Don (eds.): The Language and Sexuality Reader. London/New York, Routledge: 132140.

Aikhenvald, Alexandra Y. (2016): How Gender Shapes the World. Oxford/New York: Oxford University Press.

Aikhenvald, Alexandra Y. (2019): „Endearment, respect, and disdain through linguistic gender“. Revista Virtual de Estudos da Linguagem 17/16 (=Sonderheft: Pragmática na Gramática, ed. v. Heronides Moura). revel.inf.br/files/48a93a30dfdbc803843f22ddac4c5 41b.pdf [1.8.2020]

Azul, David (2013): „How do voices become gendered? A critical examination of everyday and medical constructions of the relationship between voice, sex, and gender identity“. In: Ah-

\footnotetext{
${ }^{29}$ Man denke nur an die sich beständig wandelnde und bislang in ihrer Komplexität noch kaum verstandene Verteilung von $d u$ und Sie seit dem Ende des 2. Weltkriegs im deutschsprachigen Raum.

${ }^{30}$ Für einen ersten Ansatz zu einer grammatisch ausgefeilten Beschreibung des Italienischen cf. jetzt Gaglia (2020)
} 
King, Malin (ed.): Challenging Popular Myths of Sex, Gender and Biology. Cham, Springer: 77-88 (= Crossroads of Knowledge 1).

Baker, Paul (2019): FABULOSA! The Story of Polari, Britain's Secret Gay Language. London: Reaktion.

Bertinetto, Pier Marco (2020): „On nominal tense “. Linguistic Typology 24: 311-352.

Bhat, D.N.S. (2004): Pronouns. Oxford/New York: Oxford University Press.

Braun, Friederike (1984): „Anredeformen im jordanischen Arabischen“. In: Winter, Werner (ed.): Anredeverhalten. Tübingen, Narr: 189-227. (= Ars Linguistica 13).

Busley, Simone/Fritzinger, Julia (in diesem Band): „Neutrales Rufnamengenus zwischen Grammatik und Pragmatik“.

Christen, Helen (1998): „Die Mutti oder das Mutti, die Rita oder das Rita? Über Besonderheiten der Genuszuweisung bei Personen- und Verwandtschaftsnamen in schweizerdeutschen Dialekten“. In: Schnyder, André et al. (eds.): Ist mir getroumet mîn leben? Vom Träumen und vom Anderssein. Festschrift für Karl-Ernst Geith zum 65. Geburtstag. Göppingen, Kümmerle: 267-281. (= Göppinger Arbeiten zur Germanistik 632).

Cicognani, Bruno (1938). „Abolizione del ,Lei““. Corriere della Sera v. 15.1.1938: 3.

Corbett, Greville G. (1991): Gender. Cambridge: Cambridge University Press.

De Vogelaer, Gunther/van der Auwera, Johan (2011): „When typological rara generate rarissima: analogical extension of verbal agreement in Dutch dialects“. In: Wohlgemuth, Jan/Cysouw, Michael (eds.): Rara \& Rarissima. Documenting the Fringes of Linguistic Diversity. Berlin/New York, Mouton de Gruyter: 47-73. (=Empirical Approaches to Language Typology 46).

Fleming, Luke (2014): „Australian exceptionalism in the typology of affinal avoidance registers“. Anthopological Linguistics 56: 115-158.

Froschauer, Regine (2003): Genus im Althochdeutschen. Eine funktionale Analyse des Mehrfachgenus althochdeutscher Substantive. Heidelberg: Winter.

Gaglia, Sascha (2020): „Hybride Kongruenz in der höflichen Anrede des Italienischen: eine diachrone Analyse“. In: Lobin, Antje/Dessì Schmid, Sarah/Fesenmeier, Ludwig (eds.): Norm und Hybridität / Ibridità e norma. Linguistische Perspektiven / Prospettive linguistiche. Berlin, Frank \& Timme: 141-167. (=Romanistik 32).

Grand, Camille (1930): ,,Tu, Voi, Lei“. Etude des pronoms allocutoires italiens. Dissertation Universität Freiburg, Schweiz. Ingenbohl: Theodose.

Greenberg, Joseph H. (1963): „Some universals of grammar with particular reference to the order of meaningful elements“. In: Greenberg, Joseph H. (ed.): Universals of Language, Cambridge (MA)/London, MIT Press: 58-90.

Grimm, Jacob (1898): Deutsche Grammatik. Vierter Theil. Besorgt durch Gustav Roethe und Edward Schröder. Gütersloh [Nachdruck: Hildesheim/Zürich/New York: Olms, 1989. (= Jacob Grimm und Wilhelm Grimm: Werke. Forschungsausgabe I, 13)].

Head, Brian F. (1978): „Respect degrees in pronominal reference“. In: Greenberg, Joseph H. (ed.): Universals of Human Language. Volume 3: Word Structure. Stanford, Stanford University Press: 151-211.

Hockett, Charles F. (1958): A Course in Modern Linguistics. New York: Macmillan.

Hübner, Julia (in diesem Band): „Genus und Sexus im Konflikt. Kongruenzformen hybrider Nomina im Sprachproduktionsprozess“. 
Hummel, Martin (2020): „Diachronic research on address in Portuguese and Spanish“. In: Hummel, Martin/Lopes, Célia dos Santos (eds.): Address in Portuguese and Spanish. Studies in Diachrony and Diachronic Reconstruction. Berlin/Boston, De Gruyter: 7-70.

Iwasaki, Shoichi/Ingkaphirom, Preeya (2005): A Reference Grammar of Thai. Cambridge: Cambridge University Press.

Jakobson, Roman ([1957]1971): „Shifters, verbal categories, and the Russian verb“. In: Ders. (1971): Selected Writings II: Word and Language. The Hague/Paris, Mouton: 130-147.

Keller, Albrecht (1904/05): „Die Formen der Anrede im Frühneuhochdeutschen“. Zeitschrift für deutsche Wortforschung 6: 129-174.

Kotthoff, Helga/Nübling, Damaris/unter Mitarbeit von Claudia Schmidt (2018): Genderlinguistik. Eine Einführung in Sprache, Gespräch und Geschlecht. Tübingen: Narr.

Ledgeway, Adam (2015): „Varieties in Italy 1“. In: Jungbluth, Konstanze/Da Milano, Federica (eds.): Manual of Deixis in Romance Languages. Berlin, Boston: De Gruyter: 75-113. (= Manuals of Romance Linguistics 6).

Leiss, Elisabeth (1997): „Genus im Althochdeutschen“. In: Glaser, Elvira/Schlaefer, Michael (eds.): Grammatica ianua artium. Festschrift für Rolf Bergmann zum 60. Geburtstag. Heidelberg, Winter: 33-48.

Listen, Paul (1999): The Emergence of German Polite Sie. Cognitive and Sociolinguistic Parameters. New York: Lang. (= Berkeley Insights in Linguistics and Semiotics 32).

Metcalf, George J. (1938): Forms of Address in German (1500-1800). St. Louis: Washington University. (= Washington University Studies N.S., Language and Literature 7).

Motschenbacher, Heiko (2007): „Can the term 'genderlect' be saved? A postmodernist re-definition". Gender and Language 1: 255-278.

Muchnik, Malka (2016): „Trying to change a gender-marked language. Classical vs. Modern Hebrew“. In: Abbou, Julie/Baider, Fabienne H. (eds.): Gender, Language and the Periphery. Grammatical and Social Gender from the Margins. Amsterdam/Philadelphia, Benjamins: $25-46$.

Nordlinger, Rachel/Sadler, Louisa (2004): „Nominal tense in crosslinguistic perspective“. Language 80: 776-806.

Nübling, Damaris/Busley, Simone/Drenda, Juliane (2013): „Dat Anna und s Eva - Neutrale Frauenrufnamen in deutschen Dialekten und im Luxemburgischen zwischen pragmatischer und semantischer Genuszuweisung“" Zeitschrift für Dialektologie und Linguistik 80: 152 196.

Nübling, Damaris et al. (2017): Historische Sprachwissenschaft des Deutschen. Eine Einführung in die Prinzipien des Sprachwandels. 5. Auflage. Tübingen: Narr.

Okamoto, Shigeko (1995): ,'Tasteless' Japanese: 'Less Feminine' Speech Among Young Japanese Women“. In: Hall, Kira/Bucholtz, Mary (eds.): Gender Articulated. Language and the Socially Constructed Self. New York, Routledge: 297-325.

Qafisheh, Hamdi (1977): A Short Referemce Grammar of Gulf Arabic. Tucson, Arizona: University of Arizona Press.

Renzi, Lorenzo (1996): „,Ma la diga, no xela venezian éla?‘ Per una storia delle forme allocutive nei dialetti italiani“. In Benincà, Paola/Cinque, Guglielmo/De Mauro, Tullio/Vincent, Nigel (eds.): Italiano e dialetti nel tempo. Saggi di grammatica per Giulio C. Lepschy. Roma, Bulzoni: 259-271. 
Ryding, Karin C. (2005): A Reference Grammar of Modern Standard Arabic. Cambridge: Cambridge University Press.

Sa'ar, Amalia (2007): „Masculine talk: On the subconscious use of masculine linguistic forms among Hebrew- and Arabic-speaking women in Israel“". Signs 32: 405-429.

Shibamoto Smith, Janet S. (2003): „Gendered structures in Japanese“. In: Hellinger, Marlis /Bußmann, Hadumod (eds.): Gender Across Languages. The Linguistic Representation of Women and Men. Vol. 3. Amsterdam/Philadelphia, Benjamins: 201-225. (=Impact 11).

Shibatani, Masayoshi (1990): The Languages of Japan. Cambridge: Camridge University Press.

Siewierska, Anna (2013): „Gender distinctions in independent personal pronouns“. In: Dryer, Matthew S./Haspelmath, Martin (eds.): The World Atlas of Language Structures Online. Leipzig: Max Planck Institute for Evolutionary Anthropology. wals.info/chapter/44 [1.8.2020].

Simon, Horst J. (2003): Für eine grammatische Kategorie ,Respekt' im Deutschen. Synchronie, Diachronie und Typologie der deutschen Anredepronomina. Tübingen: Niemeyer.

Simon, Horst J. (2010): „Zur Grammatik der indirekten Anrede im Afrikaans und im älteren Deutsch“. Germanistische Linguistik 206-209 : 395-426. (= Sonderheft: Kontrastive germanistische Linguistik, ed. v. Antje Dammel/Sebastian Kürschner/Damaris Nübling).

Simon, Horst J. (2016): „Vom Sie zum Du - und oft auch wieder zurück. Beobachtungen zur Pragmatik des temporären Anredewechsels im älteren Deutsch“. In: Bonacchi, Silvia et al. (eds.): Beziehungsgestaltung durch Sprache. Berlin, Lang: 81-85. (= Germanistik zwischen Tradition und Innovation - Akten des XIII. Internationalen Germanistenkongresses Shanghai 2015, Band 3, ed. v. Jianhua Zhu, Jin Zhao \& Michael Szurawitzki).

Steriopolo, Olga (2019): „A socio-pragmatic analysis of grammatical gender reversals“. In: Can, Cem/Patsala, Paschalia/Tatsioka, Zoi (eds.): Language in Focus: Contemporary Means and Methods in ELT and Applied Linguistics. Tallinn, LIF: 535-555.

Svennung, J. (1958): Anredeformen. Vergleichende Forschungen zur indirekten Anrede in der Dritten Person und zum Nominativ für den Vokativ. Uppsala/Wiesbaden: Almqvist \& Wiksell. (= Skrifter utgivna av K. Humanistiska Vetenskapssamfundet i Uppsala 42).

Teferra, Tsehaye/Beyl, David (1978/79): „Personal pronouns in Tigrinya: A socio-linguistic study“. Ethiopianist Notes 2/3: 61-68.

Tobin, Yishai (2001): „Gender Switch in Modern Hebrew”. In: Hellinger, Marlis/Bußmann, Hadumod (eds.): Gender Across Languages. The Linguistic Representation of Women and Men. Vol. 1. Amsterdam/Philadelphia, Benjamins: 177-198.

Tonhauser, Judith (2008): „Defining crosslinguistic categories: The case of nominal tense (Reply to Nordlinger and Sadler)“. Language 84: 332-342.

Vennemann, Theo/Wagener, Hans (1970): Die Anredeformen in den Dramen des Andreas Gryphius. München: Fink.

Weiß, Helmut (2005): „Inflected complementizers in Continental West Germanic dialects“. Zeitschrift für Dialektologie und Linguistik 72: 148-166.

Wołk, Ewa (2009): „Positive and negative emotions encoded in Amharic forms of address“. In: Pawlak, Nina (ed.): Codes and Rituals of Emotions in Asian and African Cultures. Warszawa, ELIPSA: 128-136. 
Yonezawa, Yoko (im Druck): The Mysterious Address Term anata 'You' in Japanese. Amsterdam/Philadelphia: Benjamins (= Topics in Address Research). 